\title{
Inflammation in chronic thromboembolic pulmonary hypertension: accomplice or bystander in altered angiogenesis?
}

\author{
Paul M. Hassoun \\ Affiliation: Division of Pulmonary and Critical Care Medicine, Dept of Medicine, Johns Hopkins University, \\ School of Medicine, Baltimore, MD, USA. \\ Correspondence: Paul M. Hassoun, Division of Pulmonary and Critical Care Medicine, 1830 East Monument \\ Street, Baltimore, MD 21287, USA. E-mail: phassoundjhmi.edu
}

@ERSpublications

Evidence for the role of inflammation in the pathogenesis of PAH: a new study demonstrates inflammation in CTEPH http://ow.ly/OxixF

Chronic thromboembolic pulmonary hypertension (CTEPH) is not an uncommon cause of pre-capillary pulmonary hypertension (PH). It is thought to be a long-term complication of symptomatic or asymptomatic pulmonary embolism (PE) or venous thromboembolism (VTE) [1-3]. It now appears clear from a retrospective analysis of large European registries [4] and an international prospective registry [5] that CTEPH, as its name indicates, is somehow associated with one or multiple thromboembolic events. However, the reason why a minority of patients $(\sim 0.1-9 \%)[6,7]$ who suffer an initial VTE or PE eventually develop CTEPH while the vast majority manage to resolve their clot and resume normal life remains an enigma. Some clues into the aetiology of the disease are provided by the identification of predisposing factors found in epidemiological studies [4], such as infection (osteomyelitis) and malignancy, indwelling catheters or ventriculo-atrial shunts, splenectomy, chronic inflammatory conditions (e.g. inflammatory bowel disease), and genetic predisposition involving the coagulation system $[4,6]$.

CTEPH occupies a place of predilection as the lone aetiology in group 4 of the PH classification [8], not only because of the aforementioned recognised association with VTE/PE but also because the treatment of this disease, pulmonary endarterectomy (PEA), is mainly surgical and often curative, while most other aetiologies in the $\mathrm{PH}$ classification require medical treatment with no radical cure at this time. In that context, is CTEPH really unique or does it share similarities with other pathologies that lead to $\mathrm{PH}$ ?

Inflammation has been increasingly recognised in the pathogenesis of pulmonary arterial hypertension (PAH) (group 1 of the $\mathrm{PH}$ classification), as suggested by the continuous flurry of studies and articles devoted to the subject [9-14]. In addition to the aberrant cellular proliferation (e.g. endothelial and smooth muscle cells and fibroblasts) which characterises the pulmonary vascular remodelling in PAH, influx of inflammatory cells, such as macrophages and lymphocytes, in and around the various components of the vascular wall, has long been recognised [15]. Similarly, various markers of inflammation such as cytokines and chemokines are expressed in human disease, as well as in animal models of $\mathrm{PH}$, in the lung tissue or in the circulation [16-21], and circulating antibodies have been detected [11, 22-24]. Many of these inflammatory biomarkers can predict survival [13, 25, 26].

In this issue of the European Respiratory Journal, QUARCK et al. [27] tested the hypothesis that both inflammation and deficient angiogenesis were involved in the pathogenesis of CTEPH. They specifically investigated a potential link between clinical, biological and morphometric parameters using histological

Received: June 182015 | Accepted: June 192015

Support statement: This work was supported by the U.S. Department of Health and Human Services-National Institutes of Health (NIH/NHLBI HL114910). Funding information for this article has been deposited with FundRef.

Conflict of interest: Disclosures can be found alongside the online version of this article at erj.ersjournals.com

Copyright CERS 2015 
material obtained from $52 \mathrm{CTEPH}$ patients who underwent PEA for treatment at their institution. They analysed the composition of the pathological tissues in terms of collagen, elastin, fibrin and lipid content as well as cell types (i.e. endothelial and smooth muscle cells, and fibroblasts) using immunohistochemistry as the predominant study tool. They identified four types of lesions (neointimal, thrombotic, recanalised and atherosclerotic), from which they derived a qualitative scoring system for disease severity. They observed that macrophages, T-lymphocytes and neutrophils (inflammation) accumulated essentially in atherosclerotic and thrombotic lesions, while angiogenesis was found in all four types of lesions encountered. Specimens with low scored angiogenesis predicted poor outcome, which was defined clinically as persistent pulmonary hypertension post-PEA, the need for medical therapy, and poor survival.

In a parallel assessment of circulating markers of inflammation by ELISA, they identified elevation of several inflammatory biomarkers in these patients, including C-reactive protein (CRP), interleukin-10, monocyte chemotactic protein-1, macrophage inflammatory protein- $1 \alpha$ and matrix metalloproteinase (MMP)-9, and further demonstrated that plasma CRP and MMP-9 correlated with neutrophil and macrophage accumulation, respectively. Based on these findings, the authors conclude that both inflammation and altered angiogenesis contribute to the pathogenesis of the disease and reflect poor clinical outcome.

A careful analysis of patients' clinical parameters suggested that predisposing factors or underlying diseases were present, as one might expect, in a vast majority of patients, with $77 \%$ of patients having experienced an episode of acute VTE, over a third having a thrombotic risk factor such as a lupus anticoagulant/ antiphospholipid antibody, and a significant minority of patients having another risk factor for thrombosis such as protein $\mathrm{C}$ or $\mathrm{S}$ deficiency, activated protein $\mathrm{C}$ resistance, Factor $\mathrm{V}$ Leiden mutation and antithrombin deficiency.

The multiplicity of pathological samples available to the investigators for analysis and the detailed description of the lesions, made according to the Jamieson classification, represent notable strengths of this study. Was there any surprising finding in the morphometric analysis of the specimens obtained? Regarding thrombotic lesions, while it is noted that all patients were on anticoagulation therapy for at least 3 months prior to PEA (in accordance with their local therapeutic protocol), the observation that patients who were already on PAH treatment prior to PEA were rarely found to have thrombotic lesions is perhaps not too surprising considering that most PAH drugs have some degree of antiplatelet and antithrombotic activities.

By contrast, the importance of angiogenesis in CTEPH is emphasised in this study by the fact that all patients except for one had evidence of an angiogenic process. This was determined essentially by the presence of endothelial cells or sprouting capillaries (termed the early stage of angiogenesis) and mature neovessels (late angiogenic stage) in all four lesions described, with the gradation of angiogenesis (number of neovessels) being lowest in neointimal lesions and highest in recanalised lesions. More importantly, using their scoring system the authors determined that patients with the highest angiogenic score were most likely to be long time survivors of CTEPH, while low scorers had persistent PH or required medical therapy after PEA. Similarly, "late angiogenesis" was encountered less in nonsurvivors, patients with persistent PH or those requiring medical therapy, while "early angiogenesis" was found in all CTEPH patients. In fact the combination of "overall" and "late" angiogenesis was predictive of survival, start of medical therapy and persistent $\mathrm{PH}$. These findings make intuitive sense. Less convincing, however, are the inverse correlation between overall angiogenesis and the "age of the lesion", and the finding that the "age of the lesion" was higher in nonsurvivors. Since the age of the lesion was estimated as the time lapse between the first acute episode of PE and PEA, this timing is difficult to ascertain considering that the initial clinical PE episode may not necessarily be the first thromboembolic event in these patients with predisposing factors, and nearly a quarter of the population did not have a history of VTE, hence age in their case is undetermined.

How about the role of inflammation in this study? The finding of inflammatory cells within CTEPH lesions and the presence of circulating inflammatory biomarkers are not entirely novel as they have been previously described $[28,29]$. They suggest some degree of chronic inflammation in this disease similar to $\mathrm{PAH}$. However, recruitment of neutrophils (which herald acute rather than chronic inflammation) as identified in this study is indeed intriguing. Of further interest is the presence of inflammatory cells in thrombotic and atherosclerotic, rather than neoimtimal and recanalised, lesions. This finding would suggest that inflammation might be an active participant in thrombus formation and perhaps not so important for recanalisation through proper angiogenesis. This is certainly consistent with the concept of "inflammatory thrombosis" proposed by LANG et al. [1] and others.

Finally, the authors make some interesting but rather preliminary observations that 1) vascular endothelial growth factor (VEGF) was inversely correlated with mean pulmonary arterial pressure post-PEA, and 2) low VEGF levels were found in CTEPH patients who displayed persistent PH post-PEA. These casual but intriguing observations certainly present an element of conundrum since on one hand vascular 
channels and recanalisation are thought to be driven by growth factors such as VEGF [30], and on the other hand angiogenic modulatory proteins with inflammatory properties (e.g. soluble endoglin and VEGF receptor-1) are elevated in $\mathrm{PAH}$ and predict poor outcome [25]. Importantly, from a mechanistic standpoint, the attempt by the authors to link inflammation, altered angiogenesis and clinical outcome (a tall order to be sure) falls somewhat short in this clinical study for the obvious limitations an observational clinical study presents. This link, well known to be operative in tissue repair, infectious processes and cancer, is probably driven by hypoxia and hypoxia-inducible factor-regulated signalling processes, a fundamental mechanistic underpinning that was not investigated here.

There are a few limitations in this study. Although the angiogenic score was performed by a pathologist unaware of the specific patient outcome, the scoring was qualitative rather than quantitative, not corroborated by an independent examiner, and therefore, somewhat subjective. In-hospital mortality was surprisingly high; however, the authors note in their defence that collection of the histological specimens started quite some years ago when PEA perioperative complications and death were far more common. There was also an inevitable lag between blood collection for biomarkers and histological assessment, which renders conclusions regarding morphology (with recanalisation and inflammation) and outcomes rather descriptive and speculative.

In closing, QUARCK et al. [27] clearly provide an improved and authoritative view of the landscape of CTEPH lesions and their various components. While in the realm of straight pathological observations, their rather elegant study brings new concepts about the pathogenesis of CTEPH and its clinical implications that are both highly insightful and provocative.

\section{References}

1 Lang IM. Chronic thromboembolic pulmonary hypertension - not so rare after all. $N$ Engl J Med 2004; 350: 2236-2238.

2 Dartevelle P, Fadel E, Mussot S, et al. Chronic thromboembolic pulmonary hypertension. Eur Respir J 2004; 23 : 637-648.

3 Lang I. Chronic thromboembolic pulmonary hypertension: a distinct disease entity. Eur Respir Rev 2015; 24: 246-252.

4 Bonderman D, Wilkens H, Wakounig S, et al. Risk factors for chronic thromboembolic pulmonary hypertension. Eur Respir J 2009; 33: 325-331.

5 Pepke-Zaba J, Delcroix M, Lang I, et al. Chronic thromboembolic pulmonary hypertension (CTEPH): results from an international prospective registry. Circulation 2011; 124: 1973-1981.

6 Lang IM, Pesavento R, Bonderman D, et al. Risk factors and basic mechanisms of chronic thromboembolic pulmonary hypertension: a current understanding. Eur Respir J 2013; 41: 462-468.

7 Pengo V, Lensing AW, Prins $\mathrm{MH}$, et al. Incidence of chronic thromboembolic pulmonary hypertension after pulmonary embolism. N Engl J Med 2004; 350: 2257-2264.

8 Simonneau G, Gatzoulis MA, Adatia I, et al. Updated clinical classification of pulmonary hypertension. J Am Coll Cardiol 2013; 62: Suppl., D34-D41.

9 Hautefort A, Girerd B, Montani D, et al. T-helper 17 cell polarization in pulmonary arterial hypertension. Chest 2015; 147: 1610-1620.

10 Hassoun PM, Mouthon L, Barbera JA, et al. Inflammation, growth factors, and pulmonary vascular remodeling. J Am Coll Cardiol 2009; 54: Suppl., S10-S19.

11 Nicolls MR, Taraseviciene-Stewart L, Rai PR, et al. Autoimmunity and pulmonary hypertension: a perspective. Eur Respir J 2005; 26: 1110-1118.

12 Huertas A, Tu L, Gambaryan N, et al. Leptin and regulatory T-lymphocytes in idiopathic pulmonary arterial hypertension. Eur Respir J 2012; 40: 895-904.

13 Cracowski JL, Chabot F, Labarere J, et al. Proinflammatory cytokine levels are linked to death in pulmonary arterial hypertension. Eur Respir J 2014; 43: 915-917.

14 Heresi GA, Aytekin M, Hammel JP, et al. Plasma interleukin-6 adds prognostic information in pulmonary arterial hypertension. Eur Respir J 2014; 43: 912-914.

15 Tuder RM, Groves B, Badesch DB, et al. Exuberant endothelial cell growth and elements of inflammation are present in plexiform lesions of pulmonary hypertension. Am J Pathol 1994; 144: 275-285.

16 Fartoukh M, Emilie D, Le Gall C, et al. Chemokine macrophage inflammatory protein-1alpha mRNA expression in lung biopsy specimens of primary pulmonary hypertension. Chest 1998; 114: Suppl., 50S-51S.

17 Humbert M, Monti G, Brenot F, et al. Increased interleukin-1 and interleukin-6 serum concentrations in severe primary pulmonary hypertension. Am J Respir Crit Care Med 1995; 151: 1628-1631.

18 Wright L, Tuder RM, Wang J, et al. 5-Lipoxygenase and 5-lipoxygenase activating protein (FLAP) immunoreactivity in lungs from patients with primary pulmonary hypertension. Am J Respir Crit Care Med 1998; 157: 219-229.

19 Voelkel NF, Tuder RM, Wade K, et al. Inhibition of 5-lipoxygenase-activating protein (FLAP) reduces pulmonary vascular reactivity and pulmonary hypertension in hypoxic rats. J Clin Invest 1996; 97: 2491-2498.

20 Graham BB, Chabon J, Kumar R, et al. Protective role of IL-6 in vascular remodeling in Schistosoma pulmonary hypertension. Am J Respir Cell Mol Biol 2013; 49: 951-959.

21 Steiner MK, Syrkina OL, Kolliputi N, et al. Interleukin-6 overexpression induces pulmonary hypertension. Circ Res 2009; 104: 236-244.

22 Isern RA, Yaneva M, Weiner E, et al. Autoantibodies in patients with primary pulmonary hypertension: association with anti-Ku. Am J Med 1992; 93: 307-312. 
23 Tamby MC, Chanseaud Y, Humbert M, et al. Anti-endothelial cell antibodies in idiopathic and systemic sclerosis associated pulmonary arterial hypertension. Thorax 2005; 60: 765-772.

24 Tamby MC, Humbert M, Guilpain P, et al. Antibodies to fibroblasts in idiopathic and scleroderma-associated pulmonary hypertension. Eur Respir J 2006; 28: 799-807.

25 Malhotra R, Paskin-Flerlage S, Zamanian RT, et al. Circulating angiogenic modulatory factors predict survival and functional class in pulmonary arterial hypertension. Pulm Circ 2013; 3: 369-380.

26 Soon E, Holmes AM, Treacy CM, et al. Elevated levels of inflammatory cytokines predict survival in idiopathic and familial pulmonary arterial hypertension. Circulation 2010; 122: 920-927.

27 Quarck R, Wynants M, Verbeken E, et al. Contribution of inflammation and impaired angiogenesis to the pathobiology of chronic thromboembolic pulmonary hypertension. Eur Respir J 2015; 46: 431-443.

28 Arbustini E, Morbini P, D’Armini AM, et al. Plaque composition in plexogenic and thromboembolic pulmonary hypertension: the critical role of thrombotic material in pultaceous core formation. Heart 2002; 88: 177-182.

29 Kimura $\mathrm{H}$, Okada $\mathrm{O}$, Tanabe $\mathrm{N}$, et al. Plasma monocyte chemoattractant protein-1 and pulmonary vascular resistance in chronic thromboembolic pulmonary hypertension. Am J Respir Crit Care Med 2001; 164: 319-324.

30 Waltham M, Burnand KG, Collins M, et al. Vascular endothelial growth factor and basic fibroblast growth factor are found in resolving venous thrombi. J Vasc Surg 2000; 32: 988-996. 